\title{
An Application Scenario for Single-Sided Magnetic Particle Imaging
}

K. Gräfe, Institute of Medical Engineering, University of Luebeck, Lübeck, Germany, grafe@imt.uni-luebeck.de

T. F. Sattel, Institute of Medical Engineering, University of Luebeck, Lübeck, Germany

K. Lüdtke-Buzug, Institute of Medical Engineering, University of Luebeck, Lübeck, Germany

D. Finas, Department of Obstetrics and Gynaecology, University of Luebeck, Lübeck, Germany

J. Borgert, Phillips Technology GmbH, Innovative Technologies, Research Laboratories, Hamburg, Germany

T. M. Buzug, Institute of Medical Engineering, University of Luebeck, Lübeck, Germany, buzug@,imt.uni-luebeck.de

\section{Introduction}

Magnetic Particle Imaging (MPI) is a novel tomographic imaging method. Sattel et al. first described a single-sided MPI scanner geometry. Here, sentinel lymph node biopsy for breast cancer as a medical application scenario for singlesided MPI is presented. Furthermore, simulations of different coil geometries for 2D imaging have been performed. Finally, the transfer functions of three implemented band-pass filters have been measured, confirming high spectral purity of the excitation signals.

\section{Methods}

The field-free point (FFP) applied for scanning the object of interest is obtained by superimposition of two different static magnetic fields generated by concentrically positioned coils. With an additional AC current on the inner coil, the FFP is moved in front of the scanner. Super-paramagnetic iron-oxide nanoparticles (SPIONs) are applied as tracer material. Only SPIONs in direct vicinity to the FFP contribute to the receive signal. SPIONs have a nonlinear magnetization curve, which causes a responding signal that contains the excitation frequency and its harmonics. To avoid the overlap of the particle signal with the excitation signal band-pass filters are used to keep the signal clean.

\section{Results}

Four different coil geometries and their corresponding FFP-trajectories are simulated. For 2D imaging, two coils are added to the circular coils. With one of the investigated assemblies, a rectangular field of view can be realized. In addition, the transfer functions of the band-pass filters are presented. Important for a pure excitation signal are a low damping at the excitation frequency and a high damping at the third and higher harmonics of the excitation frequency.

\section{Conclusion}

Requirements for the use of the single-sided MPI scanner for the lymph node biopsy are 2D or 3D imaging. Once the simulations are done, we find an assembly for 2D imaging and implement it. Furthermore, with the built-on filters the harmonics caused by the transmission chain are sufficiently damped and do not overlay the particle response. 